\title{
Array Concepts for Solid-State and Vacuum Microelectronics Millimeter-Wave Generation
}

\author{
RUEY J. HWU, C. F. JOU, NEVILlE C. LUHMANN, JR., M. KIM, W. W. LAM, \\ ZOYA B. POPOVIĆ, AND DAVID B. RUTLEDGE, MEMBER, IEEE
}

\begin{abstract}
There is an increasing demand for compact watt-level coherent sources in the millimeter- and submillimeter-wave region. The approach that we have taken to satisfy this need is to fabricate twodimensional grids loaded with oscillators and multipliers for quasi-optical coherent spatial combining of the outputs of large numbers of lowpower devices. This was first demonstrated through the successful fabrication of monolithic arrays with 2000 Schottky diodes. Watt-level power outputs were obtained in doubling to $66 \mathrm{GHz}$. In addition, a simple transmission-line model was verified with a quasi-optical reflectometer that measured the array impedance. This multiplier array work is being extended to novel tripler configurations employing blocking barrier devices. The technique has also been extended to oscillator configurations where the grid structure is loaded with negative-resistance devices. This was first demonstrated using Gunn devices. More recently, a 25-element MESFET grid oscillating at $10 \mathrm{GHz}$ exhibited power combining and self-locking. Currently, this approach is being extended to a 100-element monolithic array of Gunn diodes. This same approach should be applicable to planar vacuum electron devices such as the submillimeter-wave BWO and vacuum FET.
\end{abstract}

\section{INTRODUCTION}

$\mathrm{T}$ HERE is increasingly more need for watt-level sources in the $60-600 \mathrm{GHz}$ region. Although electron-beam devices such as traveling-wave tubes (TWT's), backward-wave oscillators (BWO's), and gyrotrons can readily supply the power requirements, their size, expense, and lifetime have limited their applications. The approach that we have taken is to employ solid-state microfabrication techniques to reduce the cost. To satisfy the size and lifetime constraints, we have thus far concentrated on solid-state power sources. To provide the required power levels, we have employed quasi-optical spatial power combining of the outputs of large planar arrays of devices. Here, it should be noted that this approach to

Manuscript received March 1, 1989; revised April 15, 1989

R. J. Hwu and N. C. Luhmann, Jr., are with the Department of Elec trical Engineering, University of California, Los Angeles, CA 90024.

C. F. Jou was with the Department of Electrical Engineering, University of California, Los Angeles, CA 90024. She is now with the Microwave Products Division, Hughes Aircraft Company, Torrance, CA 90509.

M. Kim, Z. B. Popović, and D. B. Rutledge are with the Division of Engineering and Applied Science, California Institute of Technology, Pasadena, CA 91125 .

W. W. Lam was with the Division of Engineering and Applied Science, California Institute of Technology, Pasadena, CA 91125. He is now with the Military Electronics Division, TRW, Redondo Beach, CA 90270.

IEEE Log Number 8929874

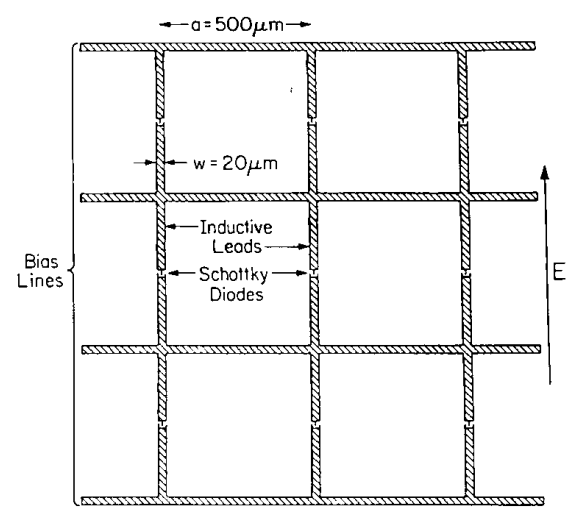

Fig. 1. Schottky-diode doubler array.

power combining significantly reduces the losses as compared to conventional waveguide approaches.

The solid-state array concept was first verified by fabricating monolithic arrays (up to several thousand elements) of Schottky diodes and antennas that served as a planar frequency multiplication surface [1]. This approach permits the use of readily available lower frequency pump sources. Recent work involves the use of more advanced blocking barrier structures to form natural tripling configurations [2]. In addition to nonlinear frequency multiplication surfaces, we have also successfully demonstrated the use of the concept to integrate a large number of negative resistance devices as a means of combining the power of solid-state oscillators [3]. This approach is attractive not only because the combining is done in free space but also because the active devices have been shown not to require an external locking signal.

In the following, we first review the above solid-state array results. We then describe possible configurations employing new vacuum electron-beam devices such as the vacuum FET [4], [5] and the planar BWO [6], [7].

\section{SchotTKy-Diode-Grid Doubler ArRay}

The concept of a monolithic diode-grid doubler array was implemented in a proof-of-principle experiment with Schottky diode grids fabricated on GaAs wafers [1]. Fig. 1 shows the grid design for the Schottky-diode doubler 


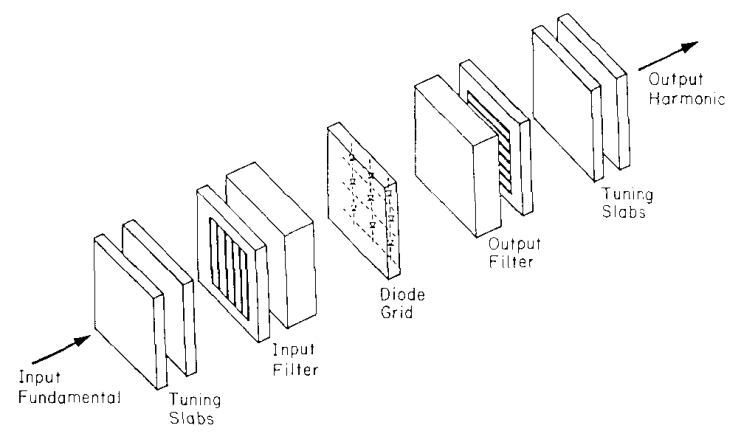

Fig. 2. Quasi-optical diode-grid doubler configuration.

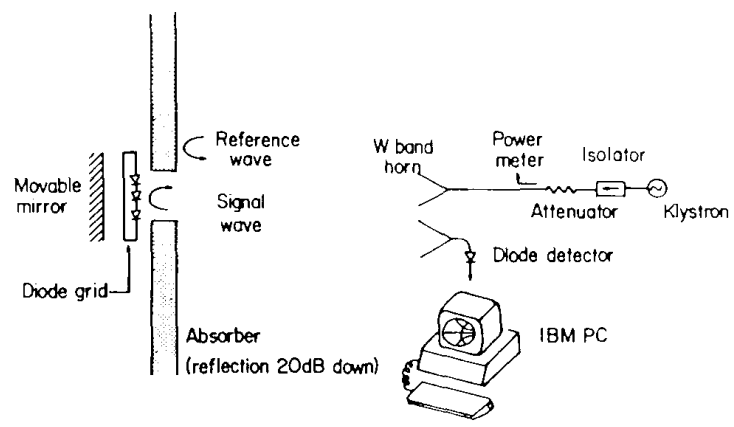

Fig. 3. Computer-controlled reflectometer for characterizing diode grids at millimeter-wave region.

array. The grid period is chosen to about half the dielectric wavelength to avoid exciting substrate modes. The vertical metal strips are inductive leads for the diodes, while the horizontal strips permit biasing the diodes. Fig. 2 schematically illustrates the quasi-optical diode-grid doubler design. Power incident normally on the grid pumps the diodes. The nonlinear capacitance of the diodes generates harmonic output power. The function of the quasi-optical filters is to select the desired harmonic. The dual dielectric slabs serve as impedance turning elements. Watt-level output power was achieved at a frequency of $66 \mathrm{GHz}$.

\section{Characterization of Diode Grid}

We have also developed a quasi-optical reflectometer technique to characterize the diode grid [8]. Referring to the experimental arrangement depicted schematically in Fig. 3, the incident beam reflects from the varactor diode grids, located in an aperture cut in the absorber. The reference beam for the computer-controlled reflectometer is obtained from the small reflection from the absorber surface. An extremely important result of the reflectometer studies was that a simple transmission-line grid model, together with the measured low-frequency parameters for the diodes, were shown to predict the measured performance over the entire capacitive bias range of the diodes, as well as over a wide range of frequencies from 33 to $141 \mathrm{GHz}$. Together with a large-signal multiplier analysis of the nonlinear varactor impedances [9], [10], this transmission line model is used to predict the multiplier performance and to facilitate detailed comparison between theory and experiment.

\section{BARrier-INTRINSIC-n ${ }^{+}$Diode-Grid Tripler ARRAY}

In an extension of the GaAs Schottky-diode doubler array studies, a back-to-back configuration of barrier-intrinsic- $n^{+}$(BIN) diodes has been developed. The BIN diode incorporates a thin (1000 $\AA$ ) undoped semiconductor layer (I) on a heavily doped layer $\left(\mathrm{n}^{+}\right)$serving as a back contact. On top of the undoped layer there is an ultrathin ( $\geq 100 \AA$ ) electron-blocking barrier layer (B) in contact with a metal top layer. This blocking layer can be formed by an insulator, a semiconductor with a very wide bandgap, or a Mott barrier. The device can be switched rapidly between two capacitance states that correspond to accumulation of electrons at the barrier and depletion of the intrinsic layer, respectively, by the applied bias. This results in a highly nonlinear capacitance-voltage characteristic that is needed for efficient harmonic generation.

Previously, we have proven the feasibility of near-millimeter-wave operation with a $\mathrm{SiO}_{2} / \mathrm{Si} \mathrm{BIN}$ diode used in a waveguide/whisker contact configuration as a frequency doubler [2]. This diode had a transit time of $1 \mathrm{ps}$ and an intrinsic cutoff frequency of $320 \mathrm{GHz}$. A maximum efficiency of 15 percent was experimentally obtained at 95 $\mathrm{GHz}$, which is in good agreement with the theoretical predictions.

Recently, a novel concept that allows the construction of a GaAs BIN diode entirely by selective doping during MBE growth (Fig. 4) has been described [2]. A Mottbarrier is formed by a thin ( $300 \AA$ ) intrinsic layer sandwiched between the top metal contact and a charge sheet created by selective doping. GaAs is superior to Si due to the higher mobility and maximum velocity, which will reduce the transit time to about $0.3 \mathrm{ps}$ and render negligible the parasitic resistance of the back contact. An intrinsic cutoff frequency of $960 \mathrm{GHz}$ can be achieved. A tripling efficiency of 35 percent at an output frequency of $100 \mathrm{GHz}$ is therefore predicted.

Due to the blocking barrier of the BIN structure, two diodes can be operated back-to-back generating a sharp symmetric spike in the capacitance-voltage curve, which eliminates even harmonics and thus favors tripler and quintupler operation. The symmetric capacitance-voltage curve from two back-to-back connected GaAs BIN diodes illustrated in Fig. 5 demonstrates the concept of the backto-back operation. The height and width of this capacitance-voltage curve can, in principle, be adjusted by doping control alone. This arrangement needs no external ohmic contact resulting in a highly efficient frequency tripler. As a further bonus, the input and output impedances are doubled.

A novel grid structure has recently been designed for the BIN diode tripler array as shown in Fig. 6. The metal grid consists of a columnar mesh of aluminum strips with 


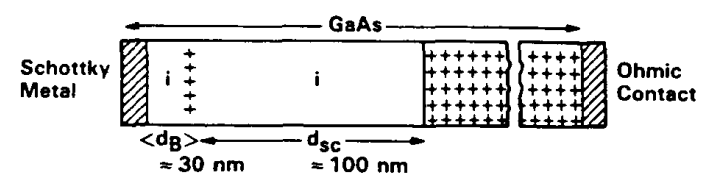

Fig. 4. Structure of the GaAs barrier-intrinsic- $n^{+}$(BIN) diode.

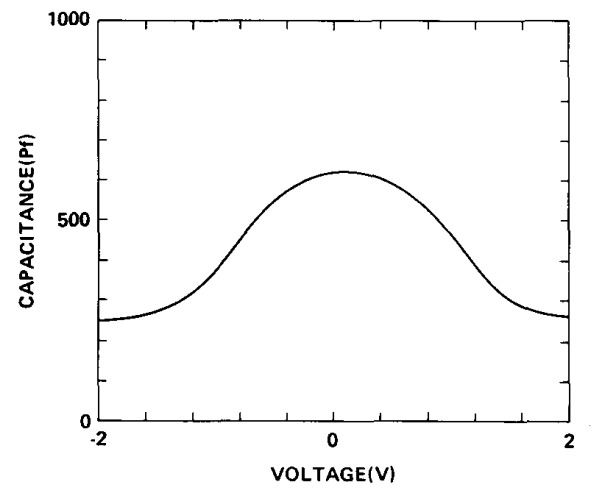

Fig. 5. Symmetric capacitance-voltage curve measured from two back-toback connected GaAs BIN diodes of the experimental device.

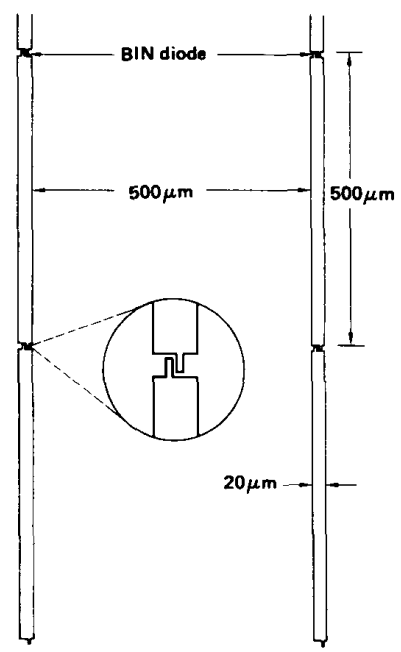

Fig. 6. BIN diode tripler array.

Schottky electrodes on each end. The small dimensions of the Schottky electrode area minimizes the zero-voltage capacitance and series resistance to increase the diode cutoff frequency. The two neighboring Schottky electrodes are designed to provide the back-to-back configuration. There is no need for dc bias lines in the diode-grid design. The grid requires only one metal pattern, which greatly facilitates the fabrication.

A quasi-optical diode-grid tripler design has been developed as illustrated in Fig. 7, where power at the fundamental frequency enters from the bottom, through an input tuner. The grating (which functions as a frequency selective filter) reflects (as a mirror) the incident pump

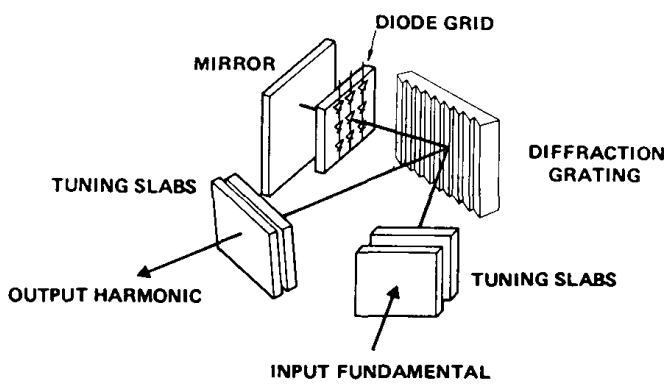

Fig. 7. Quasi-optical diode-grid tripler configuration.

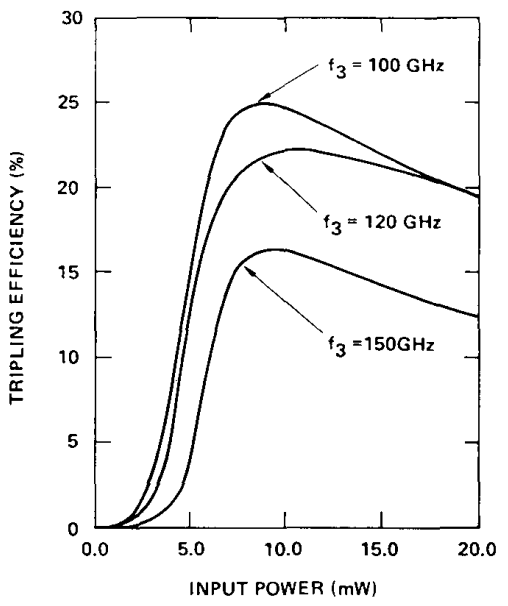

Fig. 8. Tripling efficiency versus input power at various input frequencies for the experimental GaAs BIN diode grid.

power at the fundamental frequency to the diode grid on its left, and the metal mirror behind the diode grid again reflects all the generated harmonics back to the grating plate. Harmonics are then diffracted in different directions. The third harmonic exists in the desired direction passing through an output tuner. It should be recalled that, due to the symmetric capacitance-voltage characteristics of two back-to-back connected GaAs BIN diodes, even harmonic currents cancel and even harmonic idler circuits are unnecessary thereby greatly simplifying the circuit design.

The initial BIN diode structure was grown with a conservative fabrication design (1500- $\AA$-thick epitaxial layer). This gives an intrinsic cutoff frequency of 640 $\mathrm{GHz}$. Using the arrangement of the diode-grid tripler experiment as shown in Fig. 7 for a proof-of-principle test, a watt output power at an output frequency of $100 \mathrm{GHz}$ with a tripling efficiency of 8.5 percent has been obtained from an approximately $4-\mathrm{mW}$ incident power on each diode. This experimental measurement is in good agreement with the large-signal nonlinear circuit analysis prediction (see Fig. 8). It should be mentioned that this preliminary result was performed in the low input power region. However, an optimized pumping operation promises increased performance. From the large-signal multi- 


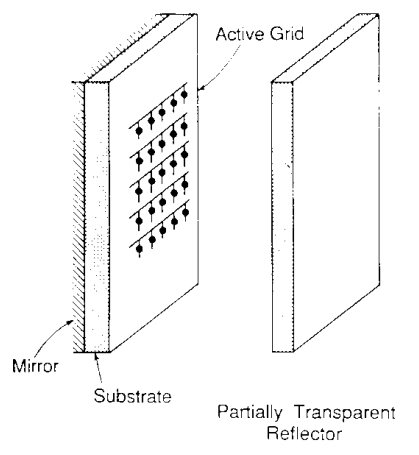

Fig. 9. Oscillator-gird resonator configuration.

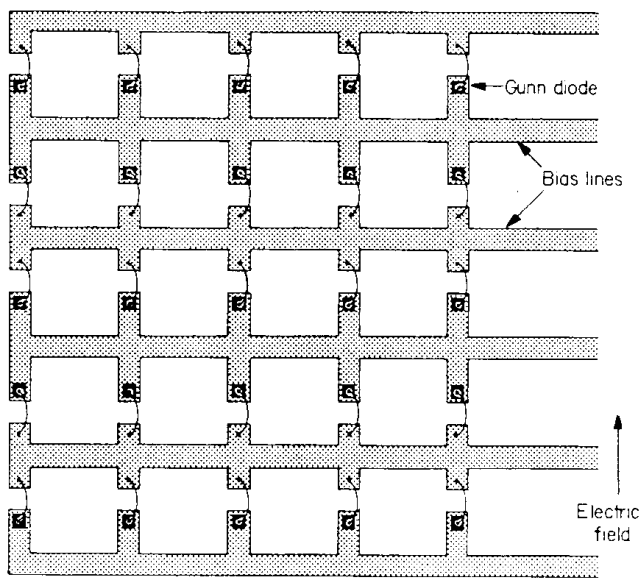

Fig. 10. Gunn diode oscillator arrray.

plier analysis results shown in Fig. 8, a maximum tripling efficiency of 24 percent at an output frequency of $100 \mathrm{GHz}$ is predicted for this particular BIN diode grid.

\section{Gunn-Diode-Grid Oscillator Array}

Power combining has been demonstrated with Gunn diodes [3] and MESFET's [11]. The oscillator configuration is shown in Fig. 9. The active grid, which is fabricated on a dielectric substrate, is placed in a transverse plane of a Fabry-Perot resonator. One of the reflectors of the resonators is partially transparent and serves as the cavity output coupler. When the devices are biased in their negative resistance region, they start oscillating at different frequencies, but the cavity provides feedback, and the devices couple through the cavity and oscillate in a single resonator mode. It is important to realize that the locking does not require an external signal. This configuration is analogous to a laser, where the active medium is the twodimensional grid, rather than the more familiar volume gain medium. Proof-of-principle measurements were performed on a 9-Gunn-diode grid [3], shown in Fig. 10. The devices exhibited self-locking and power combining between 10 and $13 \mathrm{GHz}$. The total power was found to be proportional to the square of the number of devices.

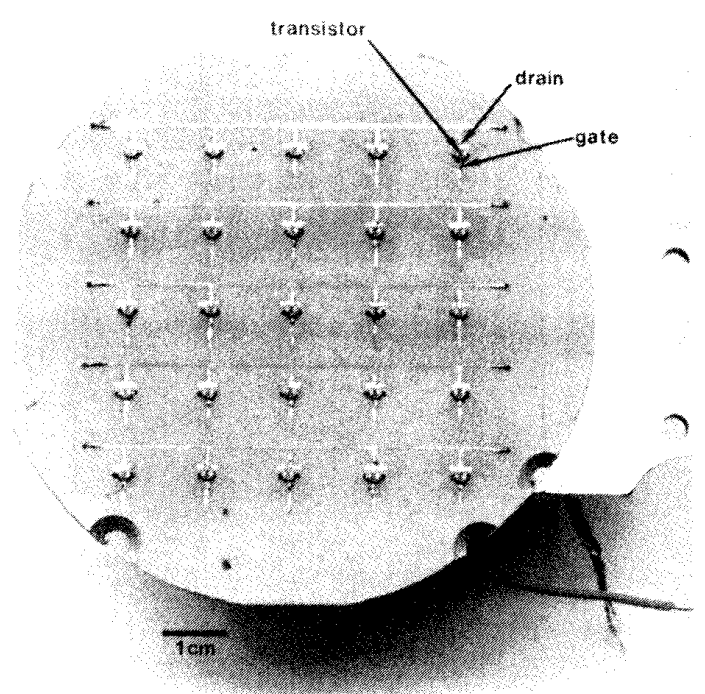

Fig. 11. Photograph of the 25-MESFET oscillator grid at $9.7 \mathrm{GHz}$.

Fig. 11 shows an $X$-band hybrid model of a 25 MESFET grid fabricated on a quarter-wavelength thick microwave substrate [11]. Simple scaling should apply for higher frequencies. The oscillator configuration is the same as in Fig. 9. The vertical lines connected to the transistor gate and drain terminals support the RF current flow, and the radiated electric field vector is parallel to them (the measured cross-polarized field was more than $20 \mathrm{~dB}$ below this polarization). The horizontal lines are used for dc bias. The maximum effective radiated power relative to an isotropic source (ERP) was $37 \mathrm{~W}$ at 9.7 $\mathrm{GHz}$. The actual radiated power from the grid was 464 $\mathrm{mW}$, which is 25 times the power typically generated with these MESFET's in single transistor microstrip circuits. The dc-to-RF conversion efficiency was 14.5 percent.

\section{Vacuum Electron-Beam Array CONFIGURATIONS}

The above described experimental studies have provided a stable foundation for the planar array concept of millimeter-wave power generation. More recently, we have begun to investigate the suitability of vacuum electron-beam devices for incorporation in planar arrays. Thus far, the two most promising concepts for such integration appear to be the vacuum FET [4], [5] and the planar submillimeter-wave BWO [6], [7]. Both of the above devices are planar and are fabricated using available solid-state processing techniques. In the following, we briefly examine their suitability for array applications.

The silicon vacuum FET in its planar realization employs an interdigitated gate-source metallization arrangement with the substrate serving as the source. This device is therefore ideal for incorporation in oscillator arrays as described above since the structure so closely resembles the familiar MESFET. Other possible modes of operation 
include a quasi-optical amplifier surface as well as a frequency multiplier making use of the device nonlinearities and negative differential resistance for high efficiency. The small interelectrode spacings result in transit times of less than $100 \mathrm{fs}$. However, before predicting arbitrarily high operating frequencies, one must account for actual input capacitance and transconductance values. For example, we have calculated that a 7500-tip thin-film field-emitter

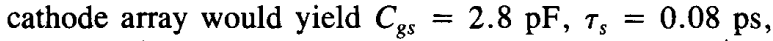
and $g_{m}=30 \mathrm{mS}$. This results in a figure of merit of $g_{m} / C_{g s}$ $=10.7 \mathrm{GHz}$. For comparison purposes, a $0.5-\mu \mathrm{m}$-gate

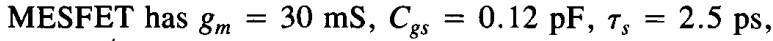
and $g_{m} / C_{g s}=250 \mathrm{GHz}$. Currently, we are investigating configurations that will improve the vacuum FET parameters and permit a vacuum FET to be used as a radiationhard millimeter-wave source.

The planar submillimeter-wave BWO concept being pursued under a NASA Lewis-University of Utah-Lincoln Laboratory collaboration [6], [7] is an ideal candidate for incorporation into planar oscillator arrays. This approach employs a interdigitated fundamental backwardwave slow-wave structure on a diamond substrate above which a planar-sheet electron-beam propagates. Operation in the fundamental spatial mode results in increased interaction impedance and reduced operating voltage as compared to the standard vane-type structures (which do not readily lend themselves to array configurations). The arrangement we conceive will closely resemble the quasioptical Gunn and FET oscillator configurations shown in Figs. 10 and 11. Here, a planar-sheet electron beam will propagate perpendicular to the surface of an array of slowwave structure attached to planar antenna structures. The remainder of the configuration will be identical to that employed for the solid-state oscillator arrays.

\section{SUMmary AND CONClusions}

Theoretical and experimental studies of both frequency multiplier and oscillator arrays have demonstrated the viability of the quasi-optical spatial power combining approach. Current directions include the study of advanced blocking barrier multiplier structures, monolithic Gunn oscillator arrays, and the use of vacuum electron-beam devices.

\section{REFERENCES}

[1] C. F. Jou et al., "Millimeter-wave monolithic Schottky diode-grid frequency doublers,"' IEEE Trans. Microwave Theory Tech., vol. 36 , no. 11, pp. $1507-1514,1988$.

[2] R. J. Hwu et al., "Watt-level millimeter-wave monolithic diode-grid frequency multipliers," Rev. Sci. Instrument, vol. 59, no. 8, pp. $1577-1579,1988$.

[3] Z. B. Popović and D. B. Rutledge, "Diode-grid oscillators," presented at the IEEE Antennas and Propagation Symp., June 1988.

[4] R. Greene, H. Gray, and B. Campisi, "Vacuum integrated circuits," in IEDM Tech. Dig., 1985, pp. 172-175.

[5] H. Gray, G. J. Campisi, and R. K. Greene, "A vacuum field effect transistor using silicon field emitter arrays," in IEDM Tech. Dig. 1986, pp. 776-779.

[6] L. Barnett, J. Baird, R. Grow, and S. Holmes, "Submillimeter-wave BWOs," in IEDM Tech Dig., 1985, pp. 364-365.
[7] 3. A. Dayton, V. O. Heien, N. Stankiewicz, and T. M. Wallett, "Submillimeter backward wave oscillators," Int. J. Infrared and Millimeter Waves, vol. 8, no. 10, pp. 1257-1268, 1987.

[8] W. W. Lam et al., "Millimeter-wave monolithic Schottky diode-grid phase shifters,"' IEEE Trans. Microwave Theory Tech., vol. 36, no. 5, pp. 902-907, 1987.

[9] D. N. Held and A. R. Kerr, "Conversion loss and noise of microwave and millimeter-wave mixers: Part 1-Theory," IEEE Trans. Microwave Theory Tech., vol, 26, pp. 49-55, 1978.

[10] H. Siegel, A. R. Kerr, and W. Hwang, "Topics in the optimization of MM wave mixers," NASA Tech. Paper 2287, 1987.

[11] Z. B. Popović, M. Kim, and D. B. Rutledge, "Grid oscillators," Int. J. Infrared and Millimeter Waves, vol. 9, no. 7, pp. 647-654, 1988.

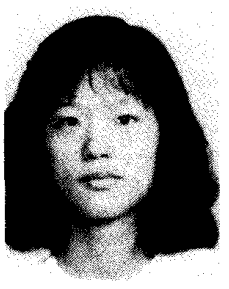

Ruey J. Hwu was born in Taipei, Taiwan. She received the M.S. degree in electrical engineering from the University of California, Los Angeles, in 1986.

Her recent research focus is in the areas of experimental measurements on and theoretical modeling of quasi-optical monolithic planar solid-state diode-grid frequency multipliers and phase shifters for use in the millimeter-wave region.

C. F. Jou, photograph and biography not available at the time of publication.

Neville C. Luhmann, Jr., received the B.S. degree in engineering physics from the University of California, Berkeley, in 1966 and the $\mathrm{Ph} . \mathrm{D}$. degree in physics from the University of Maryland, College Park, in 1972.

Following a year at the Princeton Plasma Physics Laboratory, he joined the Electrical Engineering Department at the University of California, Los Angeles, as an Assistant Professor, subsequently being promoted to Associated Professor in 1978 and Professor 1981. He is the author of more than 130 scientific papers and approximately 230 scientific presentations in the area of near-millimeter-wave imaging, gyrotrons and free-electron lasers, advanced millimeter-wave thermionic sources, near-millimeter-wave $\mathrm{Si}$ and $\mathrm{GaAs}$ sources detectors and sensors, submillimeter-wave lasers, fusion plasma diagnostics, and nonlinear electromagnetic wave-plasma interactions.

Dr. Luhmann is a member of Tau Beta Pi, Sigma Xi, and a Fellow of the American Physics Society.

tion.

W. W. Lam, photograph and biography not available at the time of publication. 


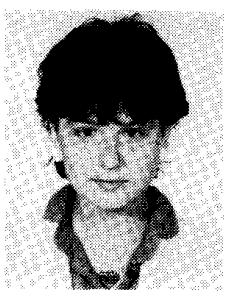

Zoya B. Popović was born in Belgrade, Yugoslavia, in 1962. She received the Dipl. Ing. degree from the University of Belgrade, Belgrade, Yugoslavia, in 1985 and the M.S. degree from the California Institute of Technology, Pasadena, in 1986. She is currently working toward the Ph.D degree at Caltech in the field of microwave- and millimeter-wave devices. Her research involves developing a semiconductor millimeter-wave highpower quasi-optical oscillator.

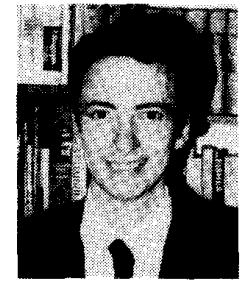

David B. Rutledge (S'75-M'80) was born in Sa vannah, GA, on January 12,1952 . He received the B.A. degree in mathematics from Williams College, Williamstown, MA, in 1973, the M.A. degree in electrical sciences from Cambridge University, England, in 1975, and the Ph.D. degree in electrical engineering from the University of California at Berkeley in 1980.

In 1980 , he joined the faculty at the California Institute of Technology, Pasadena, where he is now Professor of Electrical Engineering. His research is in developing millimeter- and submillimeter-wave monolithic integrated circuits and applications, and in software for computer-aided design and measurement. He is co-author with Prof. Richard Compton of Comell University of the software CAD program, Puff, which has over 4000 users worldwide. 